IdeAs

Idées d'Amériques

$17 \mid 2021$

Villes et culture dans les Amériques

\title{
Entre activistes et fonctionnaires : le mouvement Cultura Viva Comunitaria et les politiques culturelles à La Paz et El Alto
}

Between activists and officials: the Cultura Viva Comunitaria movement and the cultural policies in La Paz and El Alto

Entre activistas y funcionarios: el movimiento Cultura Viva Comunitaria y las políticas culturales en La Paz y El Alto

\section{Baptiste Mongis}

\section{(2) OpenEdition}

\section{Journals}

Édition électronique

URL : https://journals.openedition.org/ideas/10291

DOI : 10.4000/ideas.10291

ISSN : $1950-5701$

Éditeur

Institut des Amériques

Référence électronique

Baptiste Mongis, «Entre activistes et fonctionnaires : le mouvement Cultura Viva Comunitaria et les politiques culturelles à La Paz et El Alto », IdeAs [En ligne], 17 | 2021, mis en ligne le 01 mars 2021, consulté le 03 juin 2021. URL : http://journals.openedition.org/ideas/10291 ; DOI : https://doi.org/ 10.4000/ideas.10291

Ce document a été généré automatiquement le 3 juin 2021

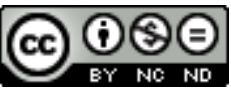

IdeAs - Idées d'Amériques est mis à disposition selon les termes de la licence Creative Commons Attribution - Pas d'Utilisation Commerciale - Pas de Modification 4.0 International. 


\title{
Entre activistes et fonctionnaires : le mouvement Cultura Viva Comunitaria et les politiques culturelles à La Paz et El Alto
}

\author{
Between activists and officials: the Cultura Viva Comunitaria movement and \\ the cultural policies in La Paz and El Alto \\ Entre activistas y funcionarios: el movimiento Cultura Viva Comunitaria y las \\ políticas culturales en La Paz y El Alto
}

Baptiste Mongis

\section{Introduction}

1 La présente étude se situe à la jonction problématique entre les revendications du mouvement Cultura Viva Comunitaria et deux initiatives récentes des municipalités de La Paz et d'El Alto, en Bolivie. Au-delà de l'apparente linéarité planifiée de l'élaboration des politiques publiques, notre but est d'identifier, comme le formule José Tasat, ces «interstices qui construisent réellement les politiques culturelles, pareils à des agencements d'éléments dissemblables, d'imprévus et de hasards issus des diverses réalités factuelles des actions culturelles $»^{1}$ (Mendes Calado P., 2015 : 9). Pour sa part, Pablo Mendes Calado dit des politiques culturelles qu'elles résultent assez peu de la simple volonté d'un décideur mais plutôt «d'un processus au cours duquel des catégories comme les intérêts sectoriels, la négociation, la bureaucratie ou les moyens, mais aussi l'imitation, les impondérables, la négligence ou les hasards ont leur lot de responsabilité » (op. cit. : 14).

2 En 2017, la ville de La Paz se dote, par le biais de sa nouvelle « Loi des Cultures », d'un programme de soutien à l'art et aux cultures, le FOCUART ${ }^{2}$, avec une ligne de financement «culture vivante communautaire ». La ville d'El Alto, pour sa part, tente 
en 2019 de donner une impulsion décisive à l'élaboration d'un texte adapté à ses problématiques locales dans le but d'obtenir à son tour sa propre Loi des Cultures dans laquelle figure également une entrée «culture vivante communautaire». Avant d'aborder ces deux cas d'étude, il nous faut brièvement rappeler les antécédents auxquels renvoie cette formule.

En 2004 débute au Brésil le programme Cultura Viva. Il est conduit par les successifs ministres de la culture Gilberto Gil et Juca Ferreira des gouvernements de Luiz Inácio «Lula » da Silva et Dilma Rousseff. Il s'ancre dans le concept des Puntos de Cultura («Points de Culture») et consiste à recenser et renforcer des espaces culturels autonomes jusque-là non reconnus par l'État. Cette politique est perçue par le militant argentin Eduardo Balán comme « la première des politiques publiques étatiques latinoaméricaines qui définit la possibilité d'incorporer la capacité populaire de construction culturelle comme outil d'un développement plus équitable sur le territoire » (Turino C., 2013 : 11). Le programme identifie, relie et soutient alors financièrement des milliers de « points de culture » communautaires éparpillés sur le territoire national, inspirant $a$ posteriori d'autres États et villes du continent.

Le directeur de théâtre Alexandre Santini, ex-fonctionnaire au ministère de la culture brésilien, souligne que si le programme brésilien Cultura Viva était le fruit d'une décision gouvernementale, le mouvement Cultura Viva Comunitaria (CVC) ${ }^{3}$ qu'il a inspiré $^{\prime}$ est l'émanation de la société civile (Santini A., 2017: 198) et notamment de ses organisations culturelles communautaires ${ }^{4}$. Il a trouvé en Colombie une impulsion décisive, en 2010, lors de la création à Medellín de la Plataforma Puente Cultura Viva Comunitaria à l'issue d'une rencontre qui a réuni une centaine d'organisations culturelles latino-américaines. Il y fut question de rechercher une autonomie politique et économique face aux projets des fondations privées et des agences de coopération, mais aussi d'acquérir «plus d'influence sur l'action publique en matière de politiques culturelles » en « établissant des ponts entre les citoyens et l'État » et en " partageant des stratégies et des responsabilités» (op. cit.: 139). Les militants du mouvement réclament dès lors $1 \%$ des budgets nationaux pour la culture et $0,1 \%$ pour la culture communautaire sur la base de législations culturelles devant être refondées «sur de nouveaux paradigmes de compréhension de la réalité et de l'action humaine » (op. cit. : 143).

5 Après s'être illustré au Sommet de la Terre de Rio en 2012 par la présence théâtrale d'une délégation bolivienne conduite par Iván Nogales et le Teatro Trono - COMPA ${ }^{5}$, le mouvement se consolide en Bolivie par la tenue d'un premier congrès en 2013, à La Paz, qui réunit à l'initiative d'organisations culturelles communautaires locales près de deux mille artistes et militants d'Amérique latine. Cet effort s'est depuis lors intensifié à l'échelle continentale (notamment par de nouveaux congrès au Salvador en 2015, en Équateur en 2017 et en Argentine en 2019) en faveur de l'élaboration de politiques culturelles «de abajo hacia arriba» (« du bas vers le haut»), comme le revendiquent les militants, c'est-à-dire depuis la logique des organisations de base vers les États et les municipalités, eux-mêmes compris comme objets de la «transformation sociale »visée par les activistes.

6 En même temps que le mouvement CVC se consolide, d'importantes municipalités, parfois associées dans des réseaux qui reconfigurent les enjeux urbains de façon prétendument « horizontale » non sans reconstituer d'autres hiérarchies (Rivière d'Arc H., 2006; Bréville B., 2020), tentent de mettre en place de nouvelles politiques 
culturelles en s'inspirant de textes-cadres internationaux. La terminologie qu'elles emploient, malgré des origines idéologiques distinctes et des divergences dans les objectifs qu'elle promeut, entre en résonance avec certaines revendications militantes qui se retrouvent prises dans ce qu'Angela Greco identifie comme le surgissement simultané de nouveaux mouvements sociaux et de l'idée de responsabilité civile entrepreneuriale (Greco A., $2008: 15)^{6}$.

7 À partir de notre expérience de terrain auprès de fonctionnaires municipaux et de coordinateurs d'organisations culturelles communautaires, nous nous proposons d'exposer ici quelques éléments d'une recherche en cours ${ }^{7}$. S'il n'est pas toujours aisé de dégager une philosophie d'action ni des objectifs clairs dans le comportement des décideurs, en particulier dans des séquences politiques instables ou électoralistes, il est toujours possible d'observer comment ils manient leurs instruments au sein de cadres institutionnels qui représentent pour eux à la fois une ressource et une contrainte (Bordat-Chauvin E., $2018: 20$ ). Il s'agira d'interroger, après avoir resitué notre contexte d'étude, le mode d'élaboration d'un programme municipal à La Paz et d'un projet de loi à El Alto en observant les interactions entre les acteurs institutionnels et les activistes et en tâchant d'analyser l'impact des politiques culturelles sur l'autonomie des organisations de base.

\section{La Paz, El Alto et l'Agenda 21 pour la culture}

Fondée en 1548, la ville coloniale de La Paz est devenue le siège du gouvernement bolivien à la fin du xix siècle. Malgré d'importantes disparités, sa population principalement métisse et blanche, plus riche et plus diplômée que celle d'El Alto, est de longue date imprégnée par des transferts culturels qui se sont particulièrement accentués ces quinze dernières années (Suárez H. J., 2018). En revanche, El Alto, vaste périphérie qui surplombe la capitale et s'étend sur l'Altiplano, n'a obtenu son statut de ville autonome qu'en 1985. Bien que ses premiers quartiers s'implantent dans les années 1940, l'espace qu'elle occupe aujourd'hui a surtout été peuplé à la fin du $\mathrm{xx}^{\mathrm{e}}$ siècle par des populations indigènes venues de communautés rurales et des familles de mineurs suite à trois vagues successives de migrations ayant engendré diverses reconfigurations identitaires et une relation spécifique avec la tradition (Poupeau F., 2010:434-447). Les villes de La Paz et El Alto, chacune dotée de près d'un million $\mathrm{d}^{\prime}$ habitants ${ }^{8}$, se jaugent autant par ce qui les différencie que par ce qui les lie l'une à l'autre. Si la capitale draine chaque jour des dizaines de milliers de travailleurs issus de sa périphérie, rendant celle-ci très dépendant du centre, la ville d'El Alto n'en dispose pas moins, et malgré un taux de chômage très élevé pour le pays (en baisse depuis les années 2000), d'un important maillage d'industries et de petites entreprises, faisant dire à Franck Poupeau qu'elle est «loin de constituer un bidonville, et se présente plutôt comme une ville ségréguée où dominent les inégalités et l'informalité au sein d'une structure corporative » (op. cit. : 438).

9 La Paz est à la fois objet de convoitise et de défiance pour les habitants de la périphérie en quête de reconnaissance. Les enquêtés s'accordent à dire que le stigmate d'habiter à El Alto persiste. Si de nombreux artistes et militants revendiquent désormais une identité alteña (d'El Alto), notamment depuis ledit « réveil indigène » et l'élection d'Evo Morales à la présidence de la Bolivie en 2005, ils n'en restent pas moins liés, entre 
critique d'un modèle et source d'inspiration, à une capitale dont ils tentent de tirer profit autant que de disputer la centralité culturelle.

Comment les décideurs publics de La Paz et d'El Alto se positionnent-ils dans un tel contexte? Comme le rappelle Élodie Bordat-Chauvin, "les responsables politicoadministratifs des politiques culturelles articulent leurs réflexions et idées sur la culture avec des définitions plus pragmatiques de leur action " (Bordat-Chauvin E., 2018 : 19). Cette articulation entre une pensée et un agir, toujours contraint, est à la fois influencée - entre autres - par les « cadres cognitifs de l'UNESCO » et les « demandes » émanant de la société civile (Ibid.).

Proposée par le chercheur australien Jon Hawkes (2001), la notion de culture comprise comme quatrième pilier du développement est valorisée en 2004 par la commission culture du réseau des Cités et Gouvernements Locaux Unis (CGLU) ${ }^{9}$ à travers l'élaboration de l'Agenda 21 de la Culture (CGLU, 2004) ${ }^{10}$ que ce réseau adopte en 2010 (CGLU, 2010). Cette articulation entre culture et développement, appuyée sur les textes de l'UNESCO, est inspirée par les travaux de Hawkes :

Pour Hawkes, la culture a toujours été question de processus davantage que de produits, ce qui implique que la valeur culturelle devient manifeste à travers sa conception et sa fabrication, bien plus qu'au travers de ce qui est le résultat $d u$ produit. Dans cette optique, l'importance de l'action culturelle, dans le cadre du développement durable, se réalise à travers les approches créatives et au sein des communautés. Sans la vitalité de la culture, la planification publique ne peut jamais être véritablement efficace ou adaptée. L'importance donnée à la fabrication de la culture, et à la place de la culture comme étant au même niveau que les autres piliers de la durabilité, souligne l'importance de la participation de la communauté, de la décentralisation et des approches qui partent de la base.

(CGLU : 2016)

Dans quelle mesure les recommandations qui insistent sur la prise en compte de la culture dans les politiques publiques trouvent-elles une incidence dans les orientations prises par des municipalités comme La Paz ou El Alto?

Parmi les conseils de la CGLU sur l'application de l'Agenda 21 de la culture, il est fait mention que le gouvernement local doit inciter à la " participation démocratique des citoyens dans la formulation, l'exercice et l'évaluation des politiques publiques de la culture» (CGLU, 2006: 3); y est notamment encouragée «la reconnaissance des différents besoins et demandes de type culturel réalisés par les personnes et les organisations d'un territoire » $(2006: 4)$.

Comment, de leur côté, les organisations de base et le mouvement Cultura Viva Comunitaria, qui œuvrent de longue date sur leurs territoires, dialoguent-ils avec de telles recommandations lorsque des décideurs s'en inspirent ou tentent de les mettre en application?

\section{Une collaboration en tension : le programme FOCUART de La Paz}

15 Le programme FOCUART fut créé en 2017 «dans le but de promouvoir des processus culturels de création, de recherche et de diffusion pour diverses manifestations culturelles, artistiques, et de gestion du patrimoine culturel $»^{11}$. En 2020, il a reçu deux cent vingt-quatre propositions, dont douze seulement dans la section « développement 
de la culture vivante communautaire " (AMN, 2020). Tâchons d'en comprendre les enjeux - les avancées comme les difficultés - tant du côté militant que du côté institutionnel.

Pour Mario Rodríguez Ibáñez ${ }^{12}$, l'un des principaux représentants du Tejido Cultura Viva Comunitaria en Bolivie ${ }^{13}$ et membre fondateur du Wayna Tambo ${ }^{14}$, le programme FOCUART est avant tout un acquis du mouvement CVC dont les organisations, à El Alto comme à La Paz, peuvent bénéficier du moment que certaines de leurs activités sont mises en place sur le territoire de la capitale. Jusque-là, affirme-t-il, seuls étaient financés « des projets ou des actions artistiques, mais jamais de processus culturels, et encore moins de base territoriale»; pour le militant, c'est une modification importante : «Si tu entres dans le programme, la municipalité s'engage quasiment à te soutenir pendant cinq ans » (Rodríguez Ibáñez M., 2020).

17 C'est en 2013, au moment de l'organisation à La Paz du premier congrès CVC, que les liens entre les organisations culturelles communautaires locales et la mairie, qui apporte un soutien logistique à la manifestation, commencent à se nouer. Selon María Cristina Garón ${ }^{15}$, responsable du programme FOCUART au moment de notre entretien, le Secrétariat des Cultures de La Paz est alors préoccupé par les questions de "déconcentration ${ }^{16}$ et de "démocratisation", mais aussi par "l'idée de culture en tant que droit » (Garón M.C., 2019). De cette façon, lorsque se met en place ledit congrès, la mairie y voit selon elle une opportunité d'alliance avec les militants afin de " générer du développement à travers la culture » (Ibid.) :

De ce congrès ont surgi ces questions autour des bénéfices de la déconcentration et de l'idée d'entendre l'art au-delà des beaux-arts, de le considérer comme un outil de transformation sociale... Et puis cette requête : que les institutions publiques devraient avoir un fonds dédié à la culture vivante communautaire. Ce fut l'occasion de créer une plate-forme, car nous avons vu que nous pouvions répondre à leur demande (Garón M. C., 2019).

18 Ces propos témoignent d'une bonne réception des revendications militantes par les pouvoirs publics. Sur l'invitation du Teatro Trono - COMPA et du Wayna Tambo, la mairie prend part à la manifestation, et un appui financier est accordé dès l'année suivante aux organisations du Tejido. C'est à ce moment que María Cristina Garón, à l'origine activiste de rue dans un projet de bibliothèque ambulante, entre en fonction au Secrétariat des Cultures. À cette époque, signale-t-elle, la mairie disposait d'un budget pour quelques services déconcentrés mais, sur le plan administratif, il était courant de contractualiser, car il n'y avait pas encore de concours. Mario Rodríguez souligne, autant que ses limites budgétaires et logistiques, les bienfaits de cette première période qui a selon lui permis de renforcer les expériences de la CVC, notamment grâce au fait que la moitié du jury qui approuvait les projets provenait du Tejido. S'il admet qu'au niveau du processus la mairie de La Paz s'est bel et bien engagée, effort qu'il salue en rappelant l'habituelle réticence des pouvoirs publics à céder de l'autonomie, il précise aussi que ce qui est né en 2014 comme un programme pour la CVC a pris une autre tournure lorsque le FOCUART a été créé en 2017 :

À ce moment-là, selon moi, nous gagnons... et nous perdons en même temps. Nous gagnons, parce que nous sommes parvenus à l'institutionnaliser: même si le gouvernement municipal change, il est tenu de maintenir FOCUART, parce que le programme et sa ligne de financement sont inscrits dans la loi. Si ce nouveau gouvernement ne lance pas la convocatoria [appel à projets], nous pouvons l'attaquer en justice [...] Mais nous perdons aussi car auparavant, lorsqu'il s'agissait encore d'un programme qui n'était pas une politique officielle, la municipalité 
devait chaque année négocier avec nous le style de fonds, où nous allions, etc. Cette modification nous a en quelque sorte mis hors-jeu en tant que mouvement. À présent, le gouvernement lance sa convocatoria sans trop se préoccuper de nous. Ce n'est plus un fonds, un programme issu de cette négociation permanente entre la mairie et le mouvement Cultura Viva Comunitaria : c'est désormais un programme officiel de l'État. ${ }^{17}$

(Rodríguez Ibáñez M., 2020)

Entrent alors en jeu deux questions : celle de la perception de la négociation à l'œuvre par des acteurs extérieurs au mouvement $\mathrm{CVC}$, et celle visant à définir qui peut bénéficier du financement intitulé "culture vivante communautaire $»^{18}$. Attentive aux rumeurs qu'elle attribue avant tout à une méconnaissance réciproque des acteurs, Maria Cristina Garón évoque celle selon laquelle le FOCUART aurait été "arrangé " entre le mouvement CVC et la municipalité dans l'intérêt du premier. Pour sa part, la fonctionnaire tend à soutenir que le programme, en prenant son indépendance vis-àvis de la demande initiale - et donc des processus de discussion avec le mouvement qui lui ont donné naissance -, a ainsi pu se démocratiser via son institutionnalisation. Le FOCUART, structuré comme une succession de financements qu'il faut obtenir dans l'ordre, s'adresse désormais à qui veut bien tenter le concours du moment que l'organisation candidate répond aux critères fixés par la mairie. Une logique qui n'a pas manqué de rencontrer quelques résistances, comme l'explique María Cristina Garón : "Ceux du Tejido voulaient, au départ, réserver cette convocatoria à ceux de leur réseau mais de notre côté, en tant que municipalité, nous nous devions de l'ouvrir à toute la société civile » (Garón M. C., 2019). Cette ouverture serait selon elle gage d'alliances, car pour atteindre le troisième palier de la ligne de financement, la "ch'aska ${ }^{19}$, qui est aussi le mieux doté, les groupes sont contraints de travailler ensemble. Valorisant cette "plate-forme » créée pour favoriser le travail d'équipe et l'émergence de nouveaux projets, la fonctionnaire en conclut que FOCUART a permis aux organisations du Tejido de s'ouvrir aux autres : avant la création de ce dispositif, ils peinaient selon elle à reconnaître le travail d'autres acteurs culturels. Ce faisant, María Cristina Garón semble chercher à résoudre trois questions à la fois : celle du soutien à des organisations associées en un mouvement dont elle reconnaît l'engagement; celle de la perception «corporatiste» de ce mouvement et de l'entre-soi de ses militants, qu'elle juge problématique ; et celle de la mise en adéquation du plan stratégique de la municipalité et de ses mécanismes de gestion culturelle avec l'Agenda 21 de la Culture, qu'elle évoque dès le début de notre entretien ${ }^{20}$. Les revendications d'autonomie et de soutien public issues «d'en bas » (le mouvement CVC) trouvent alors ici, autour de l'enjeu du FOCUART, à se frotter à des injonctions venues «d'en haut » (l'Agenda 21) qui tendent à manier un répertoire similaire. Par exemple, c'est aussi au nom des alliances, mais comprises comme une action préalable, que Mario Rodríguez critique les transformations du programme :

Les prérequis lors des précédentes convocatorias étaient d'avoir travaillé au moins trois ans dans le quartier et d'appartenir à un réseau, que ce soit le Tejido CVC ou un autre. Parce que selon nous, tu ne peux pas être un groupe de Cultura Viva Comunitaria si tu ne travailles pas en réseau, en articulation, en « tissu » : tu ne peux pas en être si tu es seul. Aujourd'hui il n'y a plus ce prérequis. Parce qu'ils [le Secrétariat des Cultures] ne perçoivent pas cette nécessité d'être en lien avec d'autres (Rodríguez Ibáñez M., 2020).

20 Les critères du « lien » diffèrent : là où le programme municipal encourage des alliances temporaires sur projet en supposant qu'elles pourraient porter leurs fruits, Mario 
Rodríguez revendique - sans nier les apports d'un programme qu'il a lui-même contribué à forger - l'idée d'un maillage pré-existant, autrement plus dense, et d'un financement pérenne au service d'un ancrage durable afin de favoriser des échanges plus profonds entre organisations et territoires. Il s'agit de défendre, pour le mouvement CVC, ce savoir-faire collectif contenu dans la notion de « tissu ». Entre 2017 et 2019, six groupes du Tejido (quatre de La Paz et deux d'El Alto), dont le Wayna Tambo, font alliance pour œuvrer dans le quartier de Cotahuma à La Paz et obtiennent, en binômes, le financement "ch'aska». Ils mettent en place un projet amené à se poursuivre avec ou sans FOCUART, précise Mario Rodríguez, car son apport financier, quoique bienvenu, reste très modeste face aux nécessités structurelles des organisations dont la capacité d'autogestion ne les rend en rien, dans le cadre de leurs activités, tributaires de cette aide ${ }^{21}$.

21 La question soulevée ici est aussi celle de la légitimité dont dispose un gouvernement pour reprendre tel quel le nom d'un mouvement dans le cadre d'un programme qu'il pilote désormais sans sa collaboration, imposant en quelque sorte son interprétation des valeurs militantes. Là où l'argument de la fonctionnaire cherche à répondre, au nom de l'intérêt général, à un souci de démocratisation de l'accès aux financements pour lutter contre des intérêts particuliers, le militant tend à décrire l'accaparement et le dévoiement par la municipalité d'un outil élaboré initialement en complicité avec les organisations. Celles-ci défendent une approche politique de la culture que tous les projets entrant désormais dans la ligne de financement «culture vivante communautaire » du FOCUART ne partagent pas spécialement : « Nous pouvons inviter n'importe qui à faire partie du Tejido, ce n'est pas un problème», clarifie Mario Rodríguez; « le souci, c'est que beaucoup de ces groupes [qui entrent dans la ligne de financement "culture vivante communautaire"] ne viennent pas du même processus de travail que nous, et donc ils ne viennent pas vers nous » (Ibid.).

L'actuel fonctionnement du FOCUART est, pour Mario Rodríguez, problématique au moins à deux titres. Le premier problème est qu'en 2019, sur les quatorze projets approuvés par la ligne de financement «culture vivante communautaire ", à peine quatre font partie du Tejido CVC, ce qui signifie que le fonds ne renforce plus spécifiquement le Tejido, mais plutôt divers projets de base communautaire. L'inconvénient est que ces derniers, selon le militant, n'arrivent pas au bout du programme (c'est-à-dire à la catégorie "ch'aska »). Le FOCUART ne contribuerait donc qu'à faire travailler ensemble ceux qui savent déjà le faire et ne parviendrait pas à initier à la politique du travail communautaire les nouveaux entrants moins expérimentés.

23 Le second problème est que le recours à la logique du concours contrevient à l'une des idées fondamentales sur lesquelles les militants avaient insisté, à savoir celle de gérer le fonds sans tomber dans une mise en concurrence : «La reconnaissance de ton travail communautaire devrait permettre que le fonds municipal te soutienne pour au moins cinq ans et te consolide. Mais ce qui s'est consolidé, c'est cette logique de compétition dans le FOCUART. C'est désormais un "fonds compétitif » [fondo concursable]. Dans ce sens-là aussi, nous avons perdu » (Ibid.).

24 Les organisations s'accommodent néanmoins du format "concours»: celles qui souhaitent se présenter consultent les autres, et les novices sont accompagnées par les plus aguerries ; un système de redistribution est aussi improvisé par les sélectionnées, en fonction des circonstances, afin de maintenir l'entraide. C'est également un format 
qui, à défaut de mieux, avait de quoi satisfaire les organisations sur certains points purement logistiques, de l'aveu de Mario Rodríguez: "Avant 2017, on nous facturait une prestation de service. Tu t'engageais à faire tant de festivals, tant de films, tant d'ateliers, tant de marchés d'artisanats. La municipalité te payait, et ce que tu faisais de cet argent ne les regardait pas » (Ibid.). Avec le concours, la logique de la « commande » disparaît : «En plus de faciliter les procédures administratives, c'est censé être encore mieux : si ta proposition gagne, tu récupères ton prix et tu en disposes librement » (Ibid.). Mais là encore, l'inconvénient tient à l'évolution du programme de financement qui pourrait bientôt échapper une fois de plus au mouvement. Dans la logique des «bonnes pratiques » encouragées par l'Agenda 21, la mairie s'orienterait vers l'idée de faire rendre des comptes aux bénéficiaires de son concours (en fléchant les dépenses et en réclamant la présentation de factures) : soit la meilleure façon, selon le co-fondateur du Wayna Tambo, d'étouffer des organisations qui ont précisément besoin d'utiliser les sommes obtenues pour des dépenses que FOCUART ne peut pas financer directement, comme les salaires, ou de les mutualiser à l'intérieur des systèmes de l'économie informelle.

\section{Une construction collective avortée : le projet de Loi des Cultures d'El Alto} Paz et de Sucre, se heurte très vite à la singularité d'El Alto. Un plan de concertation est alors mis en place et convoque plusieurs secteurs alteños liés à la culture : musiciens, danseurs, artistes du théâtre, mais également amautas et yatiris ${ }^{24}$, divers représentants de pratiques folkloriques et associations travaillant autour des cuisines autochtones, sans oublier l'Atelier d'Histoire Orale Andine (THOA) et l'Institut de Recherche en Anthropologie et Archéologie de La Paz qui intervient sur des sites à El Alto, car l'un des objectifs de la loi est aussi de développer une forme de soutien à la recherche scientifique.

Selon une convergence d'intérêts similaire à celle qui a donné naissance aux prémices du FOCUART, la volonté d'élaborer une Loi des Cultures pour la ville d'El Alto serait née d'une sollicitude du mouvement Cultura Viva Comunitaria ${ }^{22}$, mais aussi d'un intérêt qu'avait la mairie de s'en doter. Avorté pour des raisons essentiellement partisanes ${ }^{23}$, le processus de construction de la loi fut aussi compliqué par des malentendus et des divergences entre les positions institutionnelles et militantes. Le projet de loi s'est fondé sur l'idée de fortifier les centres culturels, non seulement en leur accordant un type de financement, mais aussi en générant des possibilités de renforcement et de diffusion de leurs activités, étant donné que ces espaces ne travaillent pas seulement dans le domaine artistique, et que leurs activités sont multiples.

Le processus participatif lancé en septembre 2019 est audacieux. À force d'allersretours, il doit produire une succession d'ébauches du texte de loi dont une version synthétique, après révision par des juristes, doit être remise au conseil municipal en décembre de cette même année pour une première lecture. Comme dans le cas de l'institution du programme FOCUART, il s'agit de fixer dans le droit des acquis par trop sensibles aux aléas du politique. 

Gira Angelo 25 , dit "Jimmy », est responsable de la Direction des Cultures de la ville d'El Alto au moment de notre entretien. Il affirme s'intéresser avant tout au public qui ne va pas au théâtre et nous rappelle d'emblée qu'il est avant tout artiste et directeur, depuis onze ans, d'un groupement artistique pluridisciplinaire, la Corporación Quijotada Bolivia. Recommandé auprès de la mairie par Iván Nogales du Teatro Trono - COMPA, Jaime Gira s'est temporairement mis en retrait de sa compagnie pour occuper ce poste qu'il avait d'abord décliné, réticent à se confronter aux logiques partisanes particulièrement prégnantes dans le champ politique bolivien : «J'ai accepté dans l'unique but de mettre en place cette Loi des Cultures, mais aussi la plate-forme qui permette de l'élaborer afin que les gens comprennent qu'ils ont besoin de cette loi » (Gira Angelo J., 2019). Ce défi pédagogique, déterminant pour la qualité du processus participatif censé mener à l'élaboration du texte, semble de taille. Les acteurs convoqués, selon le fonctionnaire, ne prennent pas la mesure de l'enjeu: «La culture est transversale... ça n'a rien à voir avec le fait d'aller simplement voir un spectacle. Cette loi doit pouvoir correspondre à un ensemble de nécessités fortes, présentes et vivantes, qui va de reconnaître une identité jusqu'à construire une identité propre " (Ibid.).

29 Gira et sa compagnie, qui travaillent entre La Paz et El Alto, sont entrés l'année précédente dans le Tejido CVC après plusieurs rapprochements du fait des différents postes stratégiques que le fonctionnaire a occupés ${ }^{26}$. Sa première impression du Tejido fut à l'origine sensiblement proche de celle décrite par María Cristina Garón : «On ne comprenait pas l'essence du concept Cultura Viva Comunitaria, et il circulait cette idée que c'était un groupe de gens qui s'arrangeaient pour capter des financements et les redistribuer entre eux», explique-t-il (Ibid.). À force de fréquenter les militants du mouvement, notamment lors des séances de travail pour l'élaboration de la Loi des Cultures de La Paz auquel il a participé à leurs côtés, Jaime Gira dit avoir peu à peu saisi les enjeux qui étaient les leurs. Il sollicite alors son entrée dans le Tejido et s'enquiert de consulter ses militants quand la proposition lui est faîte d'occuper ce poste de directeur du service culturel à la mairie d'El Alto. Leur réponse est enthousiaste : «Ils ont jugé bon que quelqu'un du Tejido entre là et puisse comprendre les choses depuis l'autre côté », raconte-t-il (Ibid.).

30 Alors qu'il n'est en place que depuis quelques mois, le fonctionnaire dit observer que le niveau de connaissances qu'a l'institution de ce qui se passe dans la réalité est relativement pauvre. Le fait qu'il y soit entré a contribué, selon lui, à élargir les perspectives de la municipalité en matière de politiques culturelles. Cependant, il avoue aussi s'être rendu compte, au fil du temps, que sa propre vision était assez limitée. Travailler main dans la main avec les artistes et les militants qui connaissent leur territoire est alors pour lui nécessaire :

Depuis le départ avec ce projet de loi, on a dit très clairement [depuis la mairie] qu'on ne venait pas avec une proposition déjà faite, mais au contraire, qu'on voulait écouter et savoir, et je crois que ceux qui savent le mieux sont ceux qui vivent et travaillent dans leurs quartiers, c'est-à-dire ceux de Cultura Viva Comunitaria. Mon entrée à la Direction des Cultures rend viable la volonté du mouvement CVC d'avoir une influence sur les politiques culturelles. Ce moment pendant lequel je suis un peu des deux côtés en même temps peut servir, me semble-t-il, à faire entendre ce que ces militants vivent et perçoivent comme étant des mécanismes et des normes susceptibles de satisfaire leurs besoins. Le but est de générer ensemble des espaces sur lesquels ils puissent s'appuyer et qui contribuent à ce qu'ils développent déjà sur leur territoire. 
(Gira Angelo J., 2019) Cultures d'El Alto, le rôle que s'attribuent les fonctionnaires enquêtés est, dans un premier temps au moins, similaire. Comme María Cristina Garón, Jaime Gira cherche à laisser le plus d'espace possible aux acteurs censés bénéficier des dispositifs à mettre en place : « Notre fonction est d'être la plate-forme, le pont, et non pas de "faire". Nous devons permettre que ceux qui veulent faire ou sont déjà en train de faire puissent agir dans de meilleures conditions ", explique-t-il (Ibid.). L'emploi du terme "plate-forme ", déjà présent dans le vocabulaire de la fonctionnaire de La Paz, semble ici encore se faire l'écho d'une volonté de ne pas développer à la place des organisations culturelles communautaires une conception exogène de la culture ni d'instaurer «par le haut » des dispositifs inappropriés. Mais cette rhétorique de la participation semble difficilement pouvoir répondre, sur le long terme, à la fois aux exigences normatives des «bonnes pratiques » et aux revendications d'autonomie des militants, en particulier lorsqu'elle est exploitée ou dénigrée par l'opportunisme partisan du moment. Jaime Gira déplore les innombrables difficultés qu'il a rencontré avec sa hiérarchie qui ne fut jamais réellement enthousiaste, selon lui, à l'idée de s'investir pour recueillir les demandes de la population ${ }^{27}$ (Gira Angelo J., 2021). dans les moments qui ont suivi le congrès CVC en 2013, estime que l'esprit d'ouverture de ses fonctionnaires, divisés entre partisans du maire ${ }^{28}$ et libres penseurs plus proches de l'état d'esprit du mouvement CVC, n'est globalement plus au rendez-vous (Rodriguez Ibáñez M., 2020).

Cette période de désintérêt croissant pour les revendications des organisations de la société civile coïncide avec la séquence où La Paz a présidé, de 2018 à 2019, le réseau régional des villes Mercociudades. Ce réseau, à l'origine (avec d'autres) du lancement de l'Agenda 21 de la Culture (CGLU $2004: 12$ ), est l'un de ceux qui se préoccupent, à l'échelle internationale, de former des personnels municipaux, d'encourager des échanges culturels (notamment par la circulation de troupes de théâtre et de spectacles musicaux) et de favoriser les confrontations d'expériences (Rivière d'Arc H., 2006 : 68). Si la ville d'El Alto en fait aussi officiellement partie depuis $2011^{29}$, cette adhésion est, pour Jaime Gira, toute symbolique. Expliquant avoir sollicité dans ce sens un engagement plus concret de la municipalité, il dit n'avoir obtenu auprès de sa hiérarchie qu'une évasive fin de non-recevoir. Pour lui, l'investissement dans le réseau des Mercociudades et la mise en place de l'Agenda 21 constitueraient une opportunité pour «faire circuler des artistes locaux et rendre visible la richesse des cultures autochtones » mais aussi, grâce aux échanges d'expériences, "d'apprendre des succès des autres municipalités en matière de politiques publiques » (Gira Angelo J., 2021).

Malgré le processus collaboratif mis en œuvre pour l'élaboration de la Loi des Cultures, Jaime Gira ne nie pas qu'il infléchît, par sa propre conduite en tant que directeur en poste, une certaine idée de ce que sera et ne sera pas cette loi. Il ne cache pas, par exemple, son scepticisme à l'égard du principe même de l'octroi de fonds publics :

Il peut y avoir des lignes de financement, mais si tu donnes seulement ça, tu deviens celui qui subventionne, sauf que tu ne pourras pas toujours donner de l'argent. Il ne faut pas donner de l'argent aux artistes pour qu'ils puissent travailler, il faut leur donner des moyens et des outils pour qu'ils étendent la portée de leur travail. Il ne s'agit pas de faire une loi qui nous oblige à offrir des choses, mais qui nous enseigne 
plutôt des mécanismes pour que chacun puisse générer ses propres ressources, son propre système de consommation culturelle.

(Gira Angelo J., 2019) communautaire " était, selon Jaime Gira, toujours à l'étude lorsque le projet de loi fut suspendu. Si aucun refus explicite ne fut jamais prononcé, la réticence de sa hiérarchie était grande. Celle-ci fit notamment valoir l'argument juridique : dans le cadre de la législation nationale, il était impossible d'accéder à la demande des militants, tout transfert direct de deniers publics étant illégal. Le seul moyen disponible, selon Jaime Gira, était le concours : l'instauration d'un dispositif similaire au FOCUART était d'ailleurs dans ses projets (Gira Angelo J., 2021). À l'inverse de Mario Rodríguez, Jaime Gira défend ce modèle qui, au-delà de l'intérêt de gagner, stimule selon lui l'économie locale et permet d'installer les apprentis artistes dans une dynamique de création encourageante, argument qu'il étoffe des témoignages de jeunes musiciens que lui et son équipe ont recueilli. "Certes, il existe cette logique de ne pas vouloir entrer en compétition les uns avec les autres et de tout faire en communauté, de penser en coopérative", reconnaît-il, "mais malheureusement, la compétition fait partie intégrante de notre culture, et si on ne saisit pas cette logique, on ne peut pas jouer " (Gira Angelo J., 2019).

38 Jaime Gira dit revendiquer une conception qu'il qualifie de "mixte» et dont il surmonte les contradictions en disant défendre une « industrie depuis la perspective communautaire » (Ibid.). Un modèle de politique culturelle dans lequel le fonctionnaire, 
et malgré son affinité pour la productivité de l'économie informelle alteña, défend l'idée de « faire respecter la loi [nationale], qui est la même pour tous » (Gira Angelo J., 2021). Autrement dit - et dans la ligne des craintes exprimées par Mario Rodríguez au sujet du FOCUART - celle de promouvoir des mécanismes dans lesquels il est indispensable de présenter des factures et de justifier ses dépenses. Une logique que Jaime Gira souhaite inscrire dans le cadre d'appels à projets censés être élaborés en fonction des besoins des organisations mais pilotés par la mairie; projets amenés à être financés par des deniers issus de la coopération internationale et des ambassades mais aussi des ONG, des fondations et des entreprises privées par le biais des mécanismes d'exemption d'impôts dits de « responsabilité sociale » (Ibid.).

Malgré les alliances de principe, certaines positions et propositions tendent ainsi à être discutées au sein même du Tejido CVC lors de nominations opportunes de militants à des postes institutionnels. Les réunions entre activistes et fonctionnaires s'inscrivent dès lors dans la durée contraignante de processus de délibération indexés sur l'agenda politique, ce à quoi s'ajoutent parfois des limites d'ordre juridique ou politique peu explicitées lors des discussions. Par ailleurs, il faut souligner la question toujours ouverte, pour certaines organisations de base, de leur représentativité (à savoir qui les représente et qui elles représentent), ainsi que l'investissement inégal de leurs membres dû à la précarité de leurs conditions de travail, à leur absence de compétences juridiques et à leur défiance diffuse à l'égard des processus de la politique institutionnelle. Autant de facteurs qui, dans la tenue des réunions auxquelles nous avons pu assister ou que les enquêtés nous ont rapportées, rendent très complexe la clarification des enjeux - des consensus à trouver comme des dissensus à préciser - et l'avancée des procédures.

De la même manière que le programme FOCUART de La Paz, après quelques années de fonctionnement, semble échapper aux militants qui ont contribué à le concevoir à la base, l'élaboration collaborative de la Loi des Cultures d'El Alto présente plusieurs points d'achoppement entre les activistes et l'institution dans laquelle l'un des leurs est pourtant entré.

41 Jaime Gira démissionne en février 2020 après neuf mois en poste du fait de divergences avec sa hiérarchie. Mario Rodríguez porte un regard critique sur cette collaboration avec la mairie d'El Alto et tente d'en analyser les failles. Pour lui, la tournure prise par les discussions fut très tôt décevante : «Jimmy avait dans l'idée que ce point sur le budget ne pouvait pas intégrer la loi, et que la seule chose qu'il y avait à faire était d'aller chercher directement des fonds de la coopération internationale » (Rodriguez Ibañez M., 2020). Il en conclut que Jaime Gira a agi « comme un classique gestionnaire de la culture qui ne cherchait pas une intervention politique de l'État» (Ibid.). Cela tint, selon le militant, à un malentendu qui fut fatal aux deux parties : Jaime Gira, rôdé à une logique gestionnaire entrepreneuriale distincte de celle des organisations du Tejido CVC et trop récemment entré dans le giron de ce dernier, n'aurait pas su répondre aux besoins exprimés. Pour sa part, Le Tejido n'aurait pas réussi à profiter de la présence de son nouveau partenaire à ce poste-clé et aurait échoué à clarifier avec lui ce qui, pour le mouvement, relevait de l'action des organisations de base ou de celle de l'État. La discussion ne fut pas facilitée par la conscience qu'avaient les acteurs d'avancer dans une direction difficilement concevable pour une institution qui leur a tout du long imposé ses contraintes structurelles et partisanes. 


\section{Conclusion}

Comme le soulignait en 2019 Luis Revilla, maire de La Paz, «FOCUART n'a pas seulement pour objectif de renforcer le travail de nos créateurs et gestionnaires culturels, mais aussi de générer une économie nouvelle à travers la production culturelle" (AMN, 2019a). Si une tension peut toujours exister entre les dimensions artistique et économique (selon les secteurs artistiques dont on parle et le type d'économie que l'on défend), celle qui s'invite au cœur des discussions sur les politiques culturelles étudiées ici semble surtout confronter, plus ou moins implicitement, un modèle de transformation sociale d'inspiration autonome revendiquant l'implication des pouvoirs publics, et un autre modèle plus concurrentiel, voire abstrait et technocratique, inspiré par un cahier des charges associant préoccupations économicistes normatives et modalités restreintes de participation de la société civile. Les défenseurs de l'un et de l'autre, non exempts de contradictions (du fait de leurs parcours) et travaillés par les espaces dans lesquels ils évoluent (notamment par des effets d'institution), ont en commun de vouloir s'emparer des usages de la culture (Yúdice G., 2002) comprise dans ses malléables acceptions "anthropologiques». Complexifiés par les questions relatives aux cultures communautaires et aux économies populaires, les intérêts divergents et les visées politiques des uns et des autres, malmenés de surcroît par la conjoncture, sont parfois bien incapables de s'entendre. Ces acteurs se confrontent, souvent à demi-mot et non sans tâtonnements, dans ces interstices où se discutent la création ou la gestion de programmes spécifiques et des lois censées les encadrer.

Une relation particulière, complice et défiante à la fois, s'instaure entre militants et fonctionnaires et entre mouvements et institutions, mais aussi au sein de chacun de ces espaces pris séparément. Comme le rappelle George Yúdice, la « dispute » que poursuit cet activisme culturel communautaire pour " ouvrir l'État » implique une bataille à la fois avec les acteurs qui l'occupent ou cherchent à l'occuper, et entre ceux qui cherchent à y instituer de nouvelles législations culturelles (Yúdice G., $2018: 10$ ).

Car au-delà d'un accord éventuel avec les autorités publiques, les militants de Cultura Viva Comunitaria cherchent à maintenir délibérément avec elles une forme de tension. Ce rapport paraît même être, selon l'angle d'où l'on observe, tout l'enjeu de leur lutte. Celle-ci n'est alors pas sans ambivalence. D'un côté, les changements de gouvernements, parfois radicaux en Amérique latine, ont souvent un impact décisif sur les politiques culturelles. En ce sens, les acteurs mobilisés ont tout intérêt à ce qu'un résultat de leurs revendications - quand bien même insatisfaisant - soit inscrit dans la loi : c'est d'ailleurs vers ce type de conquêtes législatives que le mouvement CVC est orienté. Cependant, et paradoxalement, les nécessités des organisations qui composent le mouvement et les divers combats qui sont les siens sont en tel décalage avec les logiques étatiques traditionnelles et la plupart des tendances gouvernementales qu'il est souvent périlleux, pour ses militants, d'établir des alliances politiques. Il leur est également difficile de s'estimer victorieux lorsque leurs objets de lutte trouvent, d'une façon ou d'une autre, à s'inscrire dans l'ordre légal. Car le changement qu'ils revendiquent, d'ordre culturel et structurel, s'appuie en partie sur des logiques économiques informelles et doit être conquis entre la préoccupation pour l'urgence et celle pour le long terme. Les activistes cherchent ici à agir sur une culture administrative et une politique économique qu'ils jugent très éloignées, d'une part, des 
besoins réels des communautés qu'ils défendent, et d'autre part, des transformations de société qu'ils revendiquent et mettent en application par leur travail artistique et culturel communautaire.

Pour employer l'expression d'Iván Nogales, il s'agit pour Cultura Viva Comunitaria de « désétatiser l'État » (Nogales I., 2019a). Autrement dit, le but est de tenter par plusieurs moyens, dans un rapport de force asymétrique mais malgré tout envisagé comme « horizontal » par les militants, d'agir sur les mécanismes de la structure étatique par le biais des acteurs qui y occupent une place et à partir de leurs interactions avec les organisations de base. La bataille menée se place donc - et de façon plus aisée lorsque l'État n'est pas aux mains d'une force politique indifférente ou ouvertement réticente au-delà de la simple recherche d'une réponse favorable à une revendication. Plus ténue, elle se situe à l'endroit même de l'espace où la discussion a lieu. Le gain de chaque lutte, lorsqu'il est obtenu, s'avère donc toujours temporaire, ambivalent, et sujet à de nouvelles discussions.

Après avoir tenté d'interpréter ces discours d'activistes et de fonctionnaires et analysé quelques disputes qu'ils occasionnent dans le champ des politiques culturelles, nous pouvons observer que, d'une part, les « recettes » de politiques urbaines importées par les réseaux de ville (Rivière d'Arc H., 2006 : 65), telle que l'Agenda 21 de la Culture, révèlent plusieurs de leurs ambivalences au contact avec le terrain. Qu'elles soient sources d'inspiration ou références explicites, leur mise en application est dans tous les cas problématique une fois confrontée à l'exigence des revendications militantes et aux préoccupations électorales locales. D'autre part, en constatant l'importance accordée par les militants au respect des processus à l'œuvre dans leurs organisations culturelles communautaires, il est difficile de ne voir dans leurs revendications qu'un simple intérêt particulier. Se dessine plutôt, dans toute sa complexité, un projet politique ancré dans son territoire et motivé par l'horizon d'une autre société, en tension avec les paradigmes venus « $d$ 'en haut » et les logiques partisanes locales.

\section{BIBLIOGRAPHIE}

Azeñas Mallea, Vanessa Tatiana, «Cultura Viva Comunitaria. Aportes y perspectivas para el Estado Plurinacional de Bolivia ", Rapport présenté pour l'obtention du titre de spécialiste en gestion de projets culturels et d'organisations d'événements, sous la direction de Danilo Oliveira, Universidade de São Paulo, Escola de comunicações e artes, Centro de estudos latinoamericanos sobre cultura e comunicação, 2015.

Bordat-Chauvin, Elodie, Les politiques culturelles en Argentine et au Mexique, Paris, L'Harmattan, 2018.

Fuentes Firmani, Emilio, « IberCultura Viva, cooperación cultural, gobierno y organizaciones », in Prato, Anna Valeria et Segura, Maria Soledad (eds.), Estado, sociedad civil y políticas culturales. Rupturas y continuidades en Argentina entre 2003 y 2017, Caseros, RGC Libros, 2018, p. 201-230. 
Greco, Angela, « Teatro comunitario en Argentina y la red Americana de Arte para el cambio social », Buenos Aires, Telón de fondo, $n^{\circ} 8,2008$.

Hawkes, Jon, The Fourth pillar of sustainability. Culture's essential role in public planning, Melbourne, Common Ground Publishing Pty Ltd, Cultural Development Network (Vic), 2001, http:// www.culturaldevelopment.net.au/community/Downloads/ HawkesJon(2001)TheFourthPillarOfSustainability.pdf, page consultée le 1er février 2021.

Mendes Calado, Pablo, Políticas culturales: rumbo y deriva. Estudio de casos sobre la (ex) Secretaría de Cultura de la Nación, Caseros, RGC libros, 2015.

Nogales Bazán, Iván, Cultura Viva Comunitaria. De más a menos, de menos a más, 2019a [non publié].

Nogales Bazán, Iván, La descolonización del cuerpo. Arte que se hace abrazo, La Paz, Vicepresidencia del Estado Plurinacional de Bolivia, 2019b.

Poupeau, Franck, « El Alto : una ficción política », La Paz, Bulletin de l'Institut Français d'Études Andines, 39 (2), 2010, p. 427-449.

Rivière d'Arc, Hélène, «La géographie et les réseaux internationaux de villes et d'urbanistes. Une vision euro-latino-américaine ", in Cahiers des Amériques latines, Des sociétés en réseaux, Paris, $\mathrm{n}^{\circ}$ 51-52, 2006, p. 63-72.

Santini, Alexandre, Cultura Viva Comunitaria. Políticas culturales en Brasil y América Latina, Caseros, RGC Libros, 2017.

Suárez, Hugo José, La Paz en el torbellino del progreso. Transformaciones urbanas en la era del cambio en Bolivia, Ciudad de México, Universidad Nacional Autónoma de México, Instituto de Investigaciones Sociales, 2018.

Turino, Celio, Puntos de cultura. Cultura viva en movimiento, Caseros, Plataforma puente Cultura Viva Comunitaria, RGC Libros, 2013.

Yúdice, George, El recurso de la cultura. Usos de la cultura en la era global, Barcelona, Editorial Gedisa, 2002.

Yúdice, George, « Prólogo », in Prato, Anna Valeria et Segura, Maria Soledad (eds.), Estado, sociedad civil y políticas culturales. Rupturas y continuidades en Argentina entre 2003 y 2017, Caseros, RGC Libros, 2018, p. 9-11.

Articles de journaux :

Bréville, Benoît, « Au nom du progressisme. Quand les grandes villes font sécession », Le Monde diplomatique, mars 2020.

Agencia Municipal de Noticias, « Alcaldía lanza II Fondo Concursable Focuart por Bs 1,5 millones para más 100 proyectos culturales », Agencia Municipal de Noticias (AMN), 12 mars 2019a, https:// amn.bo/2019/03/12/alcaldia-lanza-ii-fondo-concursable-focuart-por-bs-15-millones-paramas-100-proyectos-culturales/, page consultée le $1^{\text {er }}$ février 2021.

Agencia Municipal de Noticias, « La Alcaldía aumenta el presupuesto del FOCUART en Bs 1,7 MM para fortalecer los proyectos culturales el 2020 », Agencia Municipal de Noticias (AMN), 27 septembre 2019b, https://amn.bo/2019/09/27/la-alcaldia-aumenta-el-presupuesto-del-focuarta-bs-17-mm-para-fortalecer-los-proyectos-culturales-el-2020/, page consultée le 1er février 2021.

Página siete, « Focuart incluye asesorías para postulantes y más presupuesto », Página siete, 5 mars 2020, https://www.paginasiete.bo/cultura/2020/3/5/focuart-incluye-asesorias-parapostulantes-mas-presupuesto-248582.html, page consultée le 1er février 2021. 
Agencia Municipal de Noticias, «El FOCUART evalúa 224 postulaciones recibidas en su primera fase ", Agencia Municipal de Noticias (AMN), 24 avril 2020, https://amn.bo/2020/04/24/el-focuartevalua-224-postulaciones-recibidas-en-su-primera-fase/, page consultée le $1^{\text {er }}$ février 2021.

\section{Documents institutionnels :}

CGLU (2004) : « Agenda 21 de la culture », http://www.agenda21culture.net/sites/default/files/ files/documents/multi/ag21_fr.pdf, page consultée le $1^{\text {er }}$ février 2021.

GGLU (2006) : « Conseils sur la mise en œuvre locale de l'Agenda 21 de la culture », http:// www.agenda21culture.net/sites/default/files/files/documents/multi/docimplem_fr.pdf, page consultée le $1^{\text {er }}$ février 2021.

CGLU (2010) : « La culture : quatrième pilier du développement durable », http:// www.agenda21culture.net/sites/default/files/files/documents/fr/zz_culture4pilierdd_fra.pdf, page consultée le $1^{\text {er février } 2021 .}$

CGLU (2016) : «Prix international CGLU, Ville de Mexico, Culture 21, catégorie personnalité gagnant 2016, Jon Hawkes », http://www.agenda21culture.net/sites/default/files/files/

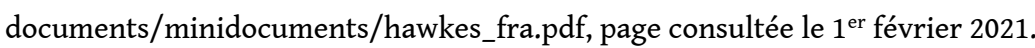

GAMLP (2017) : « Ley municipal autonómica nº 265 de fomento, salvaguarda, desarrollo y promoción de las culturas y las artes del Gobierno Autónomo Municipal de La Paz », http:// autonomias.gobernacionlapaz.com/wp-content/uploads/2015/leyes/municipal/lapaz/LMLP-265.pdf, page consultée le $1^{\text {er }}$ février 2021.

\section{Entretiens :}

Garón, María Cristina, responsable du programme FOCUART du Secrétariat aux Cultures de La Paz. Entretien réalisé le 8 novembre 2019 à La Paz.

Gira Angelo, Jaime, responsable de la Direction des Cultures de la ville d'El Alto. Entretiens réalisés le 30 octobre 2019 à El Alto et le 4 février 2021 par visioconférence.

Rodríguez Ibáñez, Mario, coordinateur culturel, représentant du Tejido Cultura Viva Comunitaria en Bolivie et membre fondateur du Wayna Tambo. Entretiens réalisés les 15 et 24 septembre 2020 par visioconférence.

\section{NOTES}

1. Toutes les traductions de l'espagnol au français sont de l'auteur.

2. Acronyme de «Fondo Concursable Municipal de Promoción al Desarrollo, Salvaguarda y Difusión de las Culturas y las Artes» (Fonds Municipal à Concourir pour la Promotion au Développement, à la Sauvegarde et à la Diffusion des Cultures et des Arts) (GAMLP, 2017).

3. Inspiré par des référents anarchistes et libertaires (Greco A., 2008 : 16) comme par l'expérience des luttes des années 1960 et 1970 (Azeñas Mallea V., 2015 : 14-16), le mouvement Cultura Viva Comunitaria est le résultat d'une articulation régionale initiée en 2003 par la création du Réseau Latino-Américain de l'Art pour la Transformation Sociale (RLATS) et renforcée par la dynamique du Forum Social Mondial de 2009 (Fuentes Firmani, E., 2018 : 204).

4. Selon la définition minimale qu'en donne Alexandre Santini - qui estime leur nombre à plus de cent-vingt mille sur tout le continent latino-américain - les organisations culturelles communautaires sont des structures qui «développement des processus culturels permanents sur leurs territoires sans être directement liées ni à la sphère publique étatique ni au marché des biens, des produits et des services culturels " (Santini A., 2017 : 124 - 127). Elles peuvent être 
aussi bien des radios communautaires que des groupes de théatre amateur, de cirque social, de rap et de hip-hop, des musées de quartier, des bibliothèques populaires ou des associations festives organisant des événements locaux.

5. Fondé en 1989 par Iván Nogales Bazán et implanté dans le quartier de Ciudad Satélite de la ville d'El Alto, le Teatro Trono - COMPA est l'articulation d'une compagnie de théâtre (le Trono) et d'un centre culturel (la Comunidad de Productores en Artes - COMPA) qui dispense dans ses locaux et dans des écoles des ateliers pédagogiques par la pratique artistique (Nogales Bazán I., 2019b). Il est l'un des principaux instigateurs du mouvement CVC à l'échelle nationale et continentale.

6. Selon Angela Greco, il s'est répandu dans le monde entrepreneurial du début des années 2000 une quête de reconnaissance définie par ladite " responsabilité d'entreprise ", conception managériale selon laquelle des entrepreneurs, «tout en répondant à leurs intérêts économiques particuliers, parviennent à générer des externalités sociales positives » (Greco A., 2018 : 16). Ces « résonances » dont nous parlons sont d'autant plus fortes que des publications et des réseaux militants dédiés à «l'art pour la transformation sociale » ont été soutenus par des fondations et des organismes internationaux maniant un vocabulaire devenu de plus en plus poreux à celui des militants.

7. Nous nous baserons sur diverses productions textuelles institutionnelles ou issues de groupes militants, ainsi que sur une sélection d'entretiens. Une dizaine d'autres, notamment avec des comédiens et des gestionnaires du Teatro Trono - COMPA, ont également enrichi cette discussion, tout comme le fait d'avoir pu assister, sur le mode d'une observation participante, à des réunions et des rencontres entre militants et fonctionnaires.

8. Les deux villes ont connu, ces dernières décennies, de très fortes poussées démographiques, mais la population d'El Alto, où les recensements sont difficilement réalisables à cause du phénomène de l'émigration saisonnière entre les campagnes et l'aire urbaine (qui ne cesse de s'étendre), est désormais supérieure à celle de La Paz dont la croissance a ralenti.

9. Créée en 2004, la CGLU serait, selon ses propres termes, «la plus grande organisation de gouvernements infranationaux au monde, avec plus de 240000 membres dans plus de 140 ÉtatsMembres de l'ONU» et entendrait favoriser, inspirée par les objectifs du Développement Durable, «l'autonomie locale démocratique [...] par la coopération entre les gouvernements locaux » (voir : https://www.uclg.org/sites/default/files/uclg_who_we_are_0.pdf).

10. Approuvé le 8 mai 2004 par des réseaux de villes, l'Agenda 21 de la culture (complément à l'Agenda 21 adopté au Sommet de la Terre de Rio en 1992) est « le premier document à vocation mondiale qui prend le pari d'établir les bases d'un engagement des villes et des gouvernements locaux en faveur du développement culturel » et notamment « dans les domaines des droits de l'Homme, de la diversité culturelle, du développement durable, de la démocratie participative et de la création de conditions pour la paix » (CGLU, $2004:$ 3).

11. Voir : https://www.facebook.com/lapazculturas/app/160430850678443

12. Entretiens réalisés les 15 et 24 septembre 2020 par visioconférence.

13. «Tejido» signifie «tissu ». Le mouvement CVC l'emploie, en Bolivie, comme synonyme de "réseau» pour se distinguer de ce dernier, jugé trop abstrait et usé dans les termes de la mondialisation capitaliste. Le mot « tejido », par sa matérialité, évoque l'étroitesse et la solidité des mailles, la proximité et la beauté des liens. Il renvoie à l'imaginaire de l'artisanat andin et des aguayos, ces toiles colorées traditionnelles. Fondé à la suite du premier congrès CVC, le Tejido bolivien précise sur sa page Facebook «ne pas être une association d'artistes mais bien un concept d'initiative socioculturelle émanant de la société civile». Voir: https:// www.facebook.com/CVCBolivia/about

14. Le Wayna Tambo est une organisation culturelle communautaire implantée depuis 1995 dans le quartier de Villa Dolores à El Alto. Notamment reconnu pour sa radio du même nom, il anime 
une revue numérique (Los Muros) et s'articule au niveau national, avec d'autres organisations, autour du Réseau de la Diversité (Red de la Diversidad).

15. Entretien réalisé le 8 novembre 2019 à La Paz.

16. À l'instar de la citation sur Jan Hawkes où était employé le terme « décentralisation » (voir supra), le mot «déconcentration" ("desconcentración») employé ici se réfère à cette même logique qui consiste en un partage de responsabilités entre les institutions publiques et les organisations de base pouvant se traduire, idéalement (pour les militants), par un soutien financier public sans ingérence, mais pouvant aussi être interprété comme une forme de désengagement ou de contrôle.

17. Ici comme souvent dans les propos des enquêtés, la notion d' «État » est confondue avec celle de "gouvernement municipal autonome": d'abord parce que ce dernier produit ses propres législations, ensuite parce que le terme «État » est employé en espagnol comme synonyme de " pouvoir public » par opposition au secteur privé et aux organisations de la société civile.

18. La Loi des Cultures de La Paz ne définit pas explicitement cette "culture vivante communautaire» et se contente, à l'article 65 , de préciser que cette section spécifique est « destinée à encourager et impulser la promotion et la diffusion de manifestations culturelles locales, d'événements et de programmes culturels de façon déconcentrée dans les différents quartiers de la municipalité » (GAMLP, 2017).

19. En quechua, « ch'aska » se réfère à l'étoile et au point le plus brillant du ciel, la planète Vénus. C'est aussi, pour Maria Cristina Garón, « comme le nœud, le point de rencontre, la tresse : quand tu tisses et que deux ou trois fils se rencontrent, ça fait une ch'aska» (Ibid.). Le référent à des mots quechuas ou aymaras et à des pratiques autochtones précolombiennes est fréquent dans les discours des militants comme dans celui des décideurs pour élaborer certains principes des politiques culturelles. María Cristina Garón revendique ainsi le principe de l'ayni, cette entraide ponctuelle par le travail collectif dans les communautés rurales, comme fondement de ce troisième palier « ch'aska » du programme FOCUART.

20. Dans la Loi des Cultures de La Paz est clairement établi que le gouvernement de la ville "prendra comme référence de ses politiques l'Agenda 21 de la Culture adopté par les Cités et Gouvernements Locaux Unis » (Chapitre III, Article 7, §3, GAMLP, 2017). La promulgation de cette loi est, comme en écho, saluée sur la page web de l'Agenda 21 comme le résultat de la mise en place de «bonnes pratiques» pour le «renforcement institutionnel, la démocratisation, la décentralisation culturelle et la revalorisation patrimoniale». Voir : http:// obs.agenda21culture.net/fr/good-practices/reconnaissance-sociale-et-projection-de-la-loimunicipale-autonome-no265-d

21. Les activités de plusieurs organisations sont rendues soutenables grâce à une dynamique solidaire avec le quartier, à la mise en place d'une monnaie d'échange placée hors de l'économie formelle, ainsi qu'à des mécanismes économiques communautaires comme le pasanaku (mutualisation continue d'une somme d'argent donnée à tour de rôle à l'un des contributeurs en fonction des besoins). Ce sont, en revanche, l'achat de matériel, les frais de maintien des lieux et la rémunération des personnes qui leur font défaut.

22. Ses militants ont été particulièrement attentifs au fait que dans la future Loi des Cultures d'El Alto devrait figurer une définition de la "cultura viva comunitaria", absente de la Loi des Cultures de La Paz. Au moment de notre enquête, la définition provisoire, sujette à d'intenses discussions, était la suivante - on notera les résonances avec le sens que Jon Hawkes accorde à la notion de culture dans la citation supra - : «Expressions communautaires privilégiant les processus aux produits, constituées en un mouvement continental de base communautaire locale, en extension permanente et voué à la convergence, et qui considère les cultures et leurs manifestations comme un bien universel et un pilier du développement humain. Ses principes fondamentaux sont l'autonomie, entendue comme aptitude à prendre des décisions, "l'esprit d'initiative» (protagonismo) et la capacitation (empoderamiento), étant donné que son action se 
focalise sur des groupes historiquement exclus des politiques et des décisions publiques. [Est aussi primordiale] l'articulation en réseau, conçue comme un dialogue des savoirs et un échange permanent d'expériences permettant de réinventer, de recréer et de rénover tant les mouvements sociaux que l'État lui-même » (Article 8, §9 de l'avant-projet municipal de « Lois des Cultures " d'El Alto) ; la définition est reprise de l'avant-projet national de "Loi-cadre des Cultures » qui n'a pas non plus abouti (Article 6, §7), et qui s'inspirait elle-même d'une formule employée dans la systématisation du premier congrès CVC en 2013.

23. Motivées par le calendrier électoral et extrapolées par la crise politique bolivienne d'octobre et novembre 2019 qui a conduit à la démission du président Evo Morales.

24. Yatiris et amautas sont à la fois des figures de médecins, de sages et de sorciers dans les traditions autochtones, les premiers se différenciant des seconds, plus anciens, par leur activité située en ville. Leurs pratiques curatrices et prédictives consistent en des rituels pour chasser les mauvais esprits, en la lecture des feuilles de coca ou en la préparation de mesas, ces plateaux composés de divers ingrédients que l'on brûle pour protéger des lieux ou les personnes lors d'un voyage ou encore garantir le succès d'une affaire ou d'une union.

25. Entretiens réalisés le 30 octobre 2019 à El Alto et le 4 février 2021 par visioconférence.

26. D'abord comme secrétaire à l'éducation de l'Association Bolivienne des Acteurs (ABDA), puis comme membre du Conseil Citoyen Interculturel de Planification des Cultures et des Arts (CONCIPCULTA).

27. La ville d'El Alto est alors dirigée par Soledad Chapetón, du parti de centre-droite Unidad Nacional, dans l'opposition au Movimiento al Socialismo (MAS) du président Evo Morales.

28. Luis Revilla, du parti de centre-gauche Sol.Bo., également dans l'opposition au MAS d'Evo Morales.

29. Voir : https://mercociudades.org/ciudades-miembro/

30. Le portefeuille de la Direction des Cultures d'El Alto pour la gestion 2019 était, selon Jaime Gira, de 8.000 .000 bolivianos, soit $0,5 \%$ du budget total de la ville d'El Alto $(1.600 .000 .000$ bolivianos en 2019). En comparaison, le portefeuille du Secrétariat des Cultures de La Paz était de 44.000.000 bolivianos (https://www.lapaz.bo/smf/dossier2019/2.1.2.html) - dont 1.500.000 destinés au programme FOCUART (AMC, 2019b) et 200.000 à la ligne de financement "cultura viva comunitaria" (Página siete, 2020) -, soit presque $2 \%$ du budget total de la ville de La Paz (2.400.000.000 bolivianos en 2019).

31. Pour Mario Rodríguez, il est à cet égard indispensable que soit préservé, à travers la mise en place de dispositifs de financement public adéquats, le complexe maillage d'une économie communautaire locale aussi riche et solidaire que fragile, basée sur des mécanismes de réciprocité, et qui ne peut souffrir l'intervention logistique directe de l'État, ayant plutôt besoin d'interfaces entre économies formelles et informelles.

\section{RÉSUMÉS}

En Bolivie, la ville autonome de La Paz s'est dotée par la "Loi des Cultures", promulguée en 2017, d'un programme de soutien à l'art et aux cultures, le FOCUART, tandis que la ville voisine d'El Alto, pour sa part, tentait en 2019 de mettre en place sa propre législation en la matière. L'une et l'autre de ces initiatives comprenaient un volet «culture vivante communautaire». Cette formule s'inscrit dans la dynamique impulsée par le mouvement Cultura Viva Comunitaria, dont le premier congrès s'est tenu en 2013 à La Paz, et qui s'est affirmé à l'échelle régionale en 
impliquant organisations culturelles communautaires, États et municipalités dans un rapport tantôt complice, tantôt en tension, tantôt marqué par une indifférence mutuelle. À travers ces deux cas d'étude, nous nous proposons d'analyser quelques-unes de ces interactions qui s'établissent entre acteurs institutionnels et militants dans l'élaboration des politiques culturelles de leurs villes.

In Bolivia, the autonomous city of La Paz, through the "Law of Cultures" promulgated in 2017, has a program to support art and culture, FOCUART, while the neighboring city of El Alto, for its part, attempted in 2019 to put in place its own legislation in this area. Both of these initiatives included a "community living culture" component. This formula is in line with the dynamics driven by the Cultura Viva Comunitaria movement, whose first congress was held in 2013 in La Paz, and which has asserted itself at the regional level by involving community cultural organizations, states and municipalities in a relationship that is sometimes complicit, sometimes tense, and sometimes marked by mutual indifference. Through these two case studies, we propose to analyze some of the interactions that take place between institutional actors and activists in the development of cultural policies in their cities.

En Bolivia, la ciudad autónoma de La Paz, a través de la "Ley de Culturas" promulgada en 2017, ha establecido un programa de apoyo al arte y la cultura, FOCUART, mientras la vecina ciudad de El Alto, por su parte, intentaba en 2019 poner en marcha su propia legislación en esta materia. Ambas iniciativas incluían un componente de "cultura viva comunitaria". Esta fórmula se inscribe en la dinámica impulsada por el movimiento Cultura Viva Comunitaria, cuyo primer congreso se celebró en 2013 en La Paz, y que se ha afirmado a nivel regional al implicar a las organizaciones culturales comunitarias, los Estados nacionales y los municipios en una relación ya sea cómplice, ya sea tensa, cuando no simplemente de indiferencia mutua. A través de estos dos estudios de caso, nos proponemos analizar algunas de las interacciones entre los actores institucionales y los activistas en el desarrollo de políticas culturales de esas ciudades.

\section{INDEX}

Palabras claves : Cultura Viva Comunitaria, políticas culturales, La Paz, El Alto, activismo

Mots-clés : Cultura Viva Comunitaria, politiques culturelles, La Paz, El Alto, activisme

Keywords : Cultura Viva Comunitaria, cultural policies, La Paz, El Alto, activism

\section{AUTEUR}

\section{BAPTISTE MONGIS}

Baptiste Mongis est diplômé d'un Master en Études théâtrales (Lyon 2), d'une licence en Sciences du langage (Paris 3) et d'un Master en Études Internationales, mention sociologie, option Amérique latine (IHEAL / Paris 3) avec un mémoire sur la mobilisation des artistes du spectacle vivant à Buenos Aires (2004 - 2017). Sa thèse porte sur les «théâtres communautaires » en Argentine et en Bolivie et le mouvement culturel latino-américain Cultura Viva Comunitaria. Il est aussi créateur lumière et régisseur pour le théâtre, professeur de pratique théâtrale, metteur en scène et auteur. 